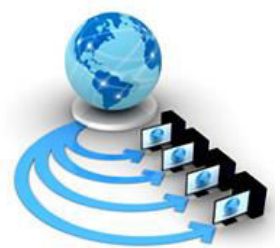

Volume 9, No. 3, May-June 2018

ISSN No. 0976-5697

International Journal of Advanced Research in Computer Science

RESEARCH PAPER

Available Online at www.ijarcs.info

\title{
COMPACT HALFMODE SIW BANDPASS FILTER FOR SUPER WIDEBAND APPLICATION
}

\author{
R.Sandhya \\ Ph.D Research Scholar, \\ Department of Electronics and Communication Engineering, \\ Sri Venkateswara College of Engineering, \\ Sriperumbudur-602117, India
}

\author{
Dr.H.Umma Habiba \\ Professor, \\ Department of Electronics and Communication Engineering, \\ Sri Venkateswara College of Engineering, \\ Sriperumbudur-602117, India
}

\begin{abstract}
In this Paper, a simple wideband BPF is proposed using a slot resonator which produces passband of 7-16 GHz. The transmission characteristics are improved by etching a square slot resonator in the half mode SIW BPF. The proposed filter uses a microstrip transition which results in better return loss performance and wider bandwidth. A novel compact bandpass filter is designed and simulated using ADS software.

Keywords- bandpass filter, wider bandwidth, pass band. transmission line is W1 and the length of the SIW
\end{abstract}

\section{INTRODUCTION}

High data rates, low power transmissions, excellent range resolution are main advantages of Ultra Wideband (UWB) technology[1]. UWB filters have lower insertion loss, good return loss performance, good selectivity and better rejection performance. A new design of wideband microwave bandpass filter is constructed by combining S-shaped slot and L-shaped slot loaded quarter-mode substrate integrated waveguide filter [2]. A bandpass filter with a $1.39 \mathrm{GHz}$ bandwidth centered at $4.54 \mathrm{GHz}$ is proposed by using CMRC resonators [4]. Good return loss performance and sharp selectivity are produced using two stubs in a bandpass filter for UWB applications [5]. Microstrip stopband bandpass filters are proposed using two shapes of split ring resonator (SRR) [6].

In this paper, half mode SIW UWB bandpass filter is realized using slot resonator. A microstrip transmission line is attached with the split ring resonator. Here, vertical array of vias are placed in the stubs of the split ring..Further optimization is done by adding $\mathrm{L}$ stub in the transmission line.

\section{DESIGN OF HMSIW BPF}

The half mode substrate integrated waveguide bandpass filter is designed in planar dielectric substrate with linear array of metallic via fences as shown in Fig.1. The SIW transmission line interrupted by two electric walls and it enables the propagation of the mode (TEm0)[7]. The parameters are the diameter of the metallic via $\mathrm{d}$, spacing between the via holes $\mathrm{p}$, the length of the transmission line is L1 and width of the

is $\mathrm{L}_{\text {SIW }}$ and width of the SIW is $\mathrm{W}_{\text {SIW. The Half mode SIW }}$ bandpass filter structure is simulated using a substrate that has a relative permittivity of 3.5 and thickness of $0.5 \mathrm{~mm}$. The resonant frequency of the pass band is derived using the following formula:

$$
f_{o}=\frac{c_{o}}{2 \varepsilon_{r}} \sqrt{\left(\frac{1}{W_{1}}\right)^{2}+\left(\frac{1}{L_{1}}\right)^{2}}
$$

Where the width and length of the transmission lines are given by the equation,

$$
W_{1}=W_{S I W}-\frac{d^{2}}{0.95 p} L_{1}=L_{S I W}-\frac{d^{2}}{0.95 p}
$$

The proposed half mode SIW bandpass filter consists of linear array of vias in the bottom. The passband bandwidth is determined by the metallic vias coupling structure. The microstrip transmission line source-load coupling structure produce dual mode and better upper stopband performance[8]. Here, coupling depends on the port microstrip transmission line. The super wide bandpass filter of passband frequency at $10 \mathrm{~dB}$ ranges from $(7-20 \mathrm{GHz})$

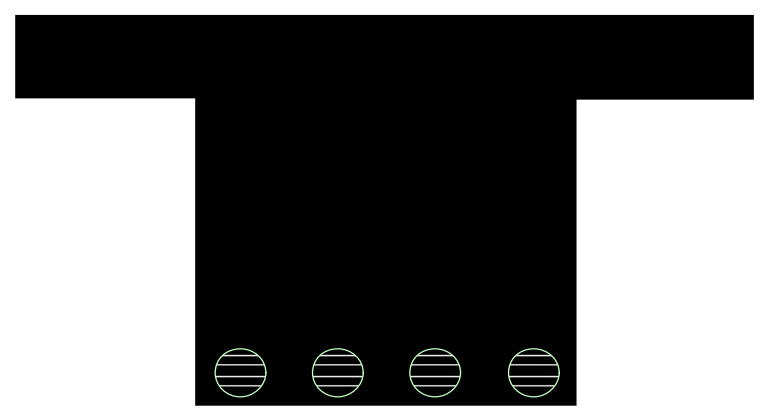

Fig.1 Configuration of Proposed UWB Bandpass filter with vertical array of vias 


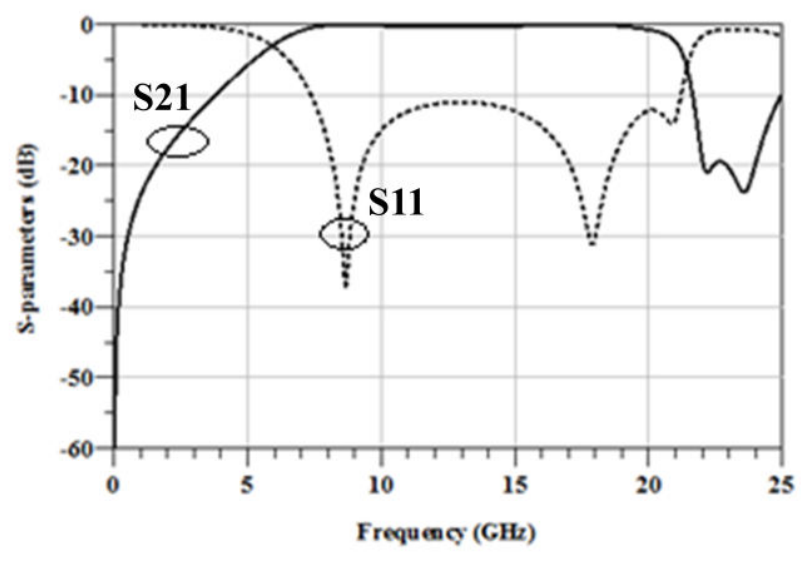

Fig.2. Simulated Result of SIW Bandpass filter using vertical array of vias.

The Surface current distribution of bandpass filter is shown in Fig3.

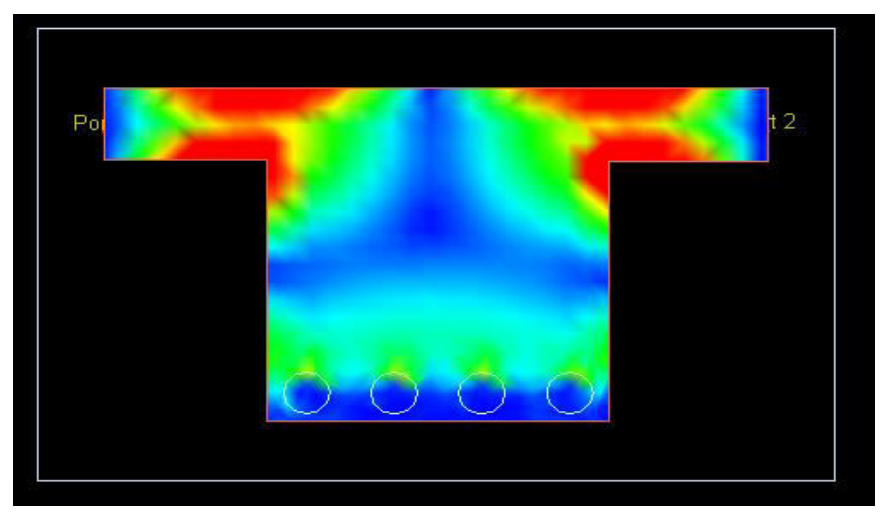

Fig.3. Surface current distributions of bandpass filter using vertical array of vias.

The analysis of compact half mode SIW bandpass filter produces a dual mode with transmission zeros[3] as shown in Fig.2. The dual mode return loss performance is improved by etching the square slot. The strong coupling depends on the size of the square slot. By decreasing the slot size, return loss and stopband performance is improved. The halfmode SIWBPF is proposed using slot resonator to provide transmission zeros in the pass band and lower insertion loss. Here via diameter is $d=0.8 \mathrm{~mm}$ and the gap between vias is $p$ is $1.5 \mathrm{~mm}$. The halfmode SIW bandpass filter structure is designed for super wide band bandpass filter frequency at $10 \mathrm{~dB}$ ranges from $(7-16 \mathrm{GHz})$.

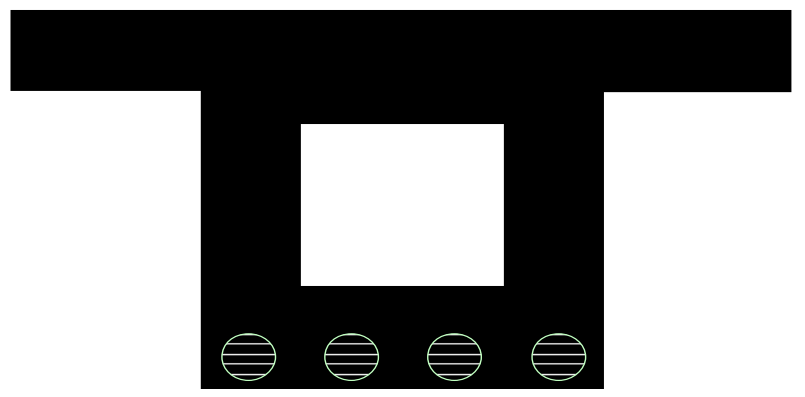

Fig.4 Configuration of Proposed UWB Bandpass filter with vertical array of vias.
The slot resonator of the half mode SIW bandpass filter produces dual mode center frequency of $8 \mathrm{GHz}$ and $14 \mathrm{GHz}$ and stopband attenuation about $-30 \mathrm{~dB}$ and obtains wide band characteristics. This halfmode SIW bandpass filter produces a super wideband bandpass filter with return loss of dual mode frequency $8 \mathrm{GHz}$ is $-40 \mathrm{~dB}$ and $14 \mathrm{GHz}$ is $-49 \mathrm{~dB}$, as shown in Fig.5.

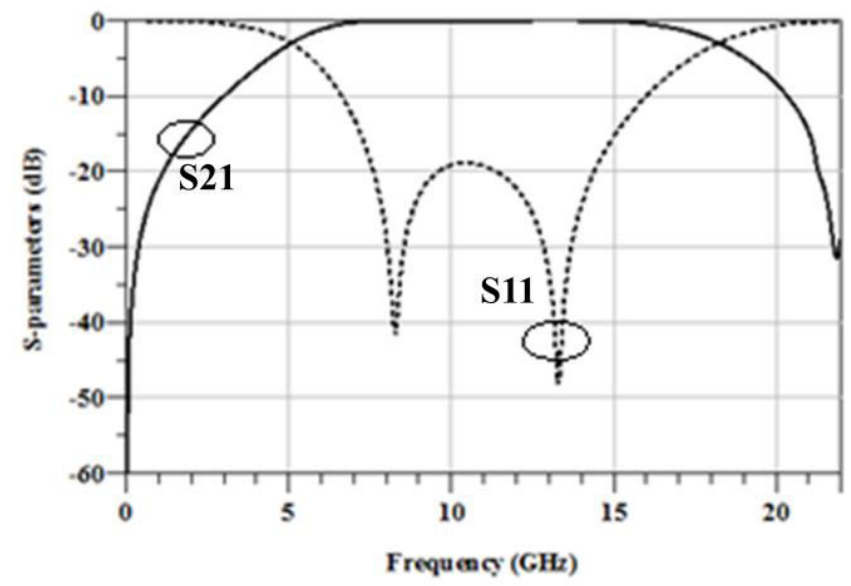

Fig.5. Simulated Result of SIW Bandpass filter using vertical array of vias.

\section{CONCLUSION}

A super wideband bandpass filter is designed and analyzed. The square slot resonator is etched in the half mode SIW bandpass filter to improve the transmission response characteristics and stopband attenuation. The half mode SIW $\mathrm{BPF}$ is designed using square slot resonator in the transmission produces return loss about -40 and $-49 \mathrm{~dB}$ and better stopband attenuation about $-30 \mathrm{~dB}$ operated frequency range of (7-16 $\mathrm{GHz}$ ).

\section{REFERENCES}

[1] FCC, Revision of Part 15, the Commission's Rules Regarding to Ultra-Wide-Band Transmission System, First Note and Order Federal Communication Commission, ET-Docket 98- 153, 2002.

[2] Wei Yang, Guo-Hui Li*, Yu-Dan Wu, Ya-Na Yang, Li Sun, and Xue-Xia Yang, "A Compact Quarter-Mode SIW Bandpass Filter with an Extremely Wide Stopband", Progress In Electromagnetics Research Letters, Vol. 67, 13-18, 2017

[3] Yu-Dan Wu, Guo-Hui Li*, Wei Yang, and Tong Mou "A Novel Dual-Band SIW Filter with High Selectivity", Progress In Electromagnetics Research Letters, Vol. 60, 81-88, 2016

[4] Lei Huang, Weiwei Wu, Xiaofa Zhang, Hongshu Lu, Yang Zhou, Naichang Yuan "A novel compact and high performance bandpass filter based on SIW and CMRC technique", International Journal of Electronics and Communication, 2017.

[5] Yong Mao Huang, Zhenhai Shao, Zhaosheng He, Chang Jiang You, and Di Jiang, A Bandpass Filter Based on Half Mode Substrate Integrated Waveguide-to-Defected Ground Structure Cell, International Journal of Antennas and Propagation,vol 2015.

[6] Zongrui He, Kaijun Song, Zihang Luo, Maoyu Fan, Yu Zhu \& Yong Fan, Compact half-mode SIW bandpass filter with highfrequency selectivity, Electromagnetics,DOI: 10.1080/02726343.2018.1436686

[7] Anil Kamma, G Shrikanth Reddy, P. Suggisetti and Jayanta Mukherjee, A Novel and Compact Ultra-Wide Band (UWB) Filter Using Modified Split Ring Resonator (MSRR), 2014 IEEE International Microwave and RF Conference (IMaRC). 
[8] Q. X. Chu, X. H. Wu, and X. K. Tian, "Novel UWB bandpass filter using stub-loaded multiple-mode resonator," IEEE
Microwave and Wireless Components Letters, vol. 21, no. 8, pp. 403-105, 2011 